\title{
Profesor Carlos Trujillo Gutiérrez: Pionero de la Electrocardiografía en Colombia
}

\section{Professor Carlos Trujillo Gutiérrez: Pioneer of electrocardiograpy in Colombia}

\author{
Alberto Barón-Castañeda
}

Cardiología, Clínica de Marly S.A., Bogotá, Colombia

Recibido el 30 de octubre de 2017; aceptado el 2 de noviembre de 2017

Disponible en Internet el 24 de diciembre de 2017

El Profesor José María Lombana Barreneche (1854 - 1928), prestigioso médico nacido en Santa Marta, es reconocido como el padre de la Medicina Interna en Colombia. Inició su ejercicio profesional en Ambalema, Tolima, y luego se radicó en Bogotá donde se vinculó con la Facultad de Medicina de la Universidad Nacional de Colombia. El doctor Lombana tuvo como alumnos a los más destacados internistas de la primera mitad del siglo xx, entre ellos el doctor Carlos Trujillo Gutiérrez, uno de sus eminentes discípulos, junto con otras personalidades de la Medicina colombiana como los doctores Alfonso Uribe Uribe, Julio Aparicio y Edmundo Rico ${ }^{1}$.

El doctor Trujillo (fig.) nació el 23 de noviembre de 1899 en Salazar de las Palmas, Norte de Santander, hijo de don José del Carmen Trujillo Ortega, importador de mercancías de Europa, y doña María Teresa Gutiérrez. Vivió en Pamplona y en 1917 se trasladó a Bogotá para adelantar estudios superiores. Ingresó a la Facultad de Medicina de la Universidad Nacional donde fue alumno de afamados profesores como los doctores José María Rivas Merizalde, Liborio Zerda, Pompilio Martínez y el ya mencionado José María Lombana Barreneche.

En la universidad fue un alumno sobresaliente y se destacó ocupando cargos como monitor en el laboratorio

Correo electrónico: albertobaronc@gmail.com de Bacteriología. En los servicios de Clínica Médica fue practicante interno de los doctores José María Lombana Barreneche y Roberto Franco. Recibió su grado de médico en 1923, con su tesis "Contribución al estudio del problema de la tuberculosis en Bogotá", para la cual contó con la asesoría del profesor Carlos Esguerra².

El médico recién graduado contrajo matrimonio con María Esther Jáuregui y del matrimonio nacieron tres hijos: Hernando, Lucía y José del Carmen. Hernando fue un cirujano egresado de la Universidad Nacional, pionero de la anestesiología inhalada en Colombia. Luego ejerció su profesión en los Estados Unidos. José del Carmen es un ilustre internista formado en la Universidad Nacional, Miembro Fundador de la Asociación Colombiana de Medicina Interna, en 1958.

El doctor Trujillo viajó a Europa donde estudió Medicina Clínica durante dos años, en el Hôtel Dieu de Paris, el Hospital más antiguo de la capital francesa ${ }^{3}$. Tenía especial interés en la Clínica Médica; en el Hospital Laennec tuvo la oportunidad de escuchar las famosas Conferencias Clínicas del profesor Louis Ramond. Fue alumno de importantes profesores como Ferdinand Widal (1862-1929), estudioso de la fiebre tifoidea y desarrollador de la prueba diagnóstica por aglutinación, y Louis Henry Vaquez (1860 -1936), cardiólogo francés quien introdujo la electrocardiografía y el registro de pulso carotideo en su país. Recordemos que Vaquez 


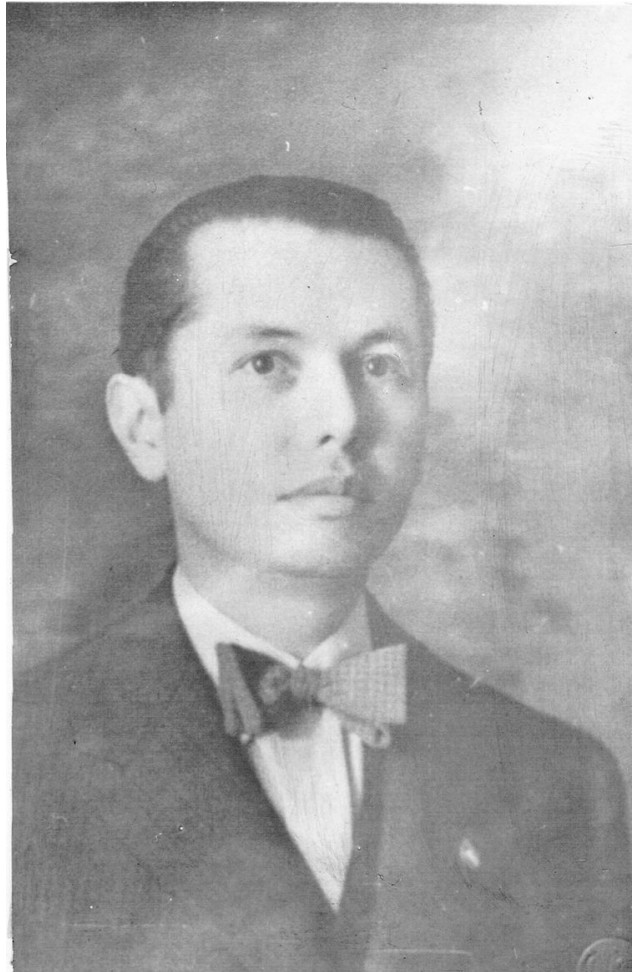

Figura Fotografía del doctor Carlos Trujillo Gutiérrez. Cortesía del Doctor José del Carmen Trujillo Jáuregui.

estudió junto con William Osler la policitemia vera o enfermedad de Vaquez-Osler ${ }^{4}$.

En 1925 regresó a Colombia y trajo consigo novedosos equipos médicos entre los que se destaca el primer electrocardiógrafo que llegó al país, un equipo francés marca Boulitte. Esto ocurrió pocos años después de la invención del electrocardiógrafo realizada por el médico Willem Einthoven nacido en Semarang, isla de Java, entonces colonia holandesa ${ }^{5,6}$. Para dar a conocer su invento en 1901,publicó "Un nouveau galvanometre" y en 1903 "Die galvanometrische Registrierung des menschlichen Elektrokardiogramms", 7. Por su creación y por la descripción de las ondas P, QRS y T del electrocardiograma recibió el premio Nobel en $1924^{8}$.

La toma del electrocardiograma entonces era un proceso muy laborioso. Se trataba de electrocardiógrafo de hilo de plata y para la inscripción el rodillo se tiznaba y sobre esto quedaba el trazado de los micro-voltajes generados por el corazón. Apenas se obtenía se llevaba el registro a un fotógrafo quien hacía el trabajo de revelado y entregaba el material gráfico para la interpretación. Era poco lo que se conocía entonces de la electrocardiografía, pero basado en sus observaciones, el doctor Trujillo planteó la hipótesis que el registro electrocardiográfico es diferente en la fase aguda de un infarto de miocardio del trazado que se obtiene algún tiempo después. En su aparato trabajaron el profesor J. Hernando Ordóñez Garay y Luis Guillermo Forero Nougés, pioneros de la Cardiología colombiana.

También trajo al país aparatos de rayos $\mathrm{x}$, de diatermia, microscopio y elementos de laboratorio. Esta importación le dio un gran impulso hacia la modernización a la medicina colombiana?.
Desde su regreso a Bogotá ejerció la Medicina interna e inició su carrera docente en la Universidad Nacional. Por sus profundos conocimientos y por su interés por la materia, fue el responsable de la cátedra de Fisiología desde 1929 hasta 1938. En 1935 ganó por concurso la posición de profesor agregado de Clínica Médica y de la misma manera obtuvo el título de profesor titular en 1939.

La enseñanza de la Medicina Clínica se hacía entonces al estilo francés, en dos años. El primero dedicado a la Patología Interna; tenía a su disposición el Pabellón San Carlos del Hospital San Juan de Dios y contaba con dos profesores agregados, dedicados al tema; entre ellos estaban Francisco Gnecco Mozo, considerado el padre de la Endocrinología en Colombia, y Rafael Martín Rodríguez. Había cercanía entre profesores y alumnos y se realizaba enseñanza tutorial en los pabellones del Hospital San Juan de Dios. El Jefe de Clínica (equivalente al residente de hoy) o el interno presentaba la historia clínica a los profesores y finalmente el profesor Trujillo examinaba al paciente y discutía en detalle los hallazgos semiológicos, el diagnóstico diferencial, su fisiopatología y tratamiento. Algunos de los Jefes de Clínica a su cargo fueron Pablo Elías Gutiérrez en 1945, Rafael Carrizosa Argáez en 1947, Ricardo Cediel Ángel y Roberto de Zubiría Consuegra; más tarde todos fueron profesores en la Universidad. Así mismo, fue profesor de Biología en el Externado y de Medicina Clínica en la Universidad Javeriana ${ }^{3}$.

Gracias a sus amplios conocimientos de fisiología y su capacidad de deducción, fue precursor del concepto actual de la apoptosis en la insuficiencia cardiaca: cuando le presentaron un paciente con falla cardiaca en estado terminal en el análisis del caso dijo a sus alumnos: "Por la hipoxia permanente, en el cardíaco hay muerte de las células todos los días. Por eso, cuando el cardíaco muere, no sólo deja de vivir sino también de morir" "2.

Desde su regreso de Paris el doctor Trujillo ingresó a la Academia Nacional de Medicina y pocos años después, en 1931, asumió el cargo de Tesorero de la corporación, cuando el presidente era el doctor Zoilo Cuéllar Durán; también actuó interinamente como secretario. Fue reelegido Tesorero para el período 1932 a 1936, durante la presidencia del doctor Rafael Ucrós ${ }^{9,10}$.

Además de su formación médica y de su capacidad docente se destacó por su obra científica. Desde muy joven realizó publicaciones. Después de entregar su tesis de grado sobre la tuberculosis, durante los años 1923 a 1932 publicó varias observaciones fisiológicas sobre "Las arritmias de tensión o anisonofonogramas"'. En 1932 publicó un estudio de un caso clínico de endocarditis maligna de evolución lenta (enfermedad de Osler) ${ }^{11}$, patología poco conocida en esa época en Colombia. De acuerdo con su pensamiento científico y capacidad de análisis, publicó en la revista de la Universidad Nacional "Juicio crítico sobre un trabajo científico" "12, donde resalta cómo se debe analizar en forma objetiva un estudio clínico. Publicó así mismo, temas diversos como: "El tratamiento quirúrgico de la tuberculosis en Bogotá", "Estudio sobre Higiene y Asistencia Pública", "Acción tóxica de la avertina sobre el centro respiratorio", "Estudios fisiológicos sobre la arritmia de tensión", "Estenosis del intestino delgado por peritonitis bacilar" y "Estudio clínico del paludismo", "3. Analizó en detalle "El trabajo del corazón en Bogotá. Introducción al estudio de la tensión 
arterial media en Colombia"13 presentado en la Academia Nacional de Medicina por el doctor Francisco Gnecco Mozo al concurso "Manuel Forero".

Fue un analítico de las diferentes modalidades de fiebre y su posible relación con el diagnóstico. Como algo relacionado con este tema se interesó en el paludismo y fue un defensor de la teoría de la enfermedad sistémica propuesta por el doctor Antonio Vargas Reyes, en contradicción de la teoría esplénica que dominaba a finales del siglo XIX y comienzos del $X^{14}$. El Doctor Trujillo planteó la teoría fluxionaria capilar con la idea que la fluxión se podía producir en cualquier órgano y la sintomatología dependía de su localización ${ }^{13}$. En 1943 publicó sus observaciones sobre el Paludismo en la Revista de la Facultad de Medicina de la Universidad Nacional ${ }^{15}$.

Falleció en Bogotá el 24 de agosto de 1960 como consecuencia de la ruptura de un aneurisma cerebral.

\section{Conflicto de intereses}

Ninguno.

\section{Agradecimientos}

Al doctor José del Carmen Trujillo Jáuregui, por sus importantes aportes a la biografía y por la fotografía del Profesor.

Al doctor Hugo Sotomayor Tribín, por su colaboración con bibliografía.

\section{Bibliografía}

1. D’Achiardi R. Conferencia Lombana Barreneche, La Medicina Interna en Colombia. Relevancia de la Asociación Colombiana de Medicina Interna. Acta Médica Colombiana. 2009;34:42-7.

2. De Zubiría R, Cala RA. Semblanza de un Profesor. Carlos Trujillo Gutiérrez. Acta Médica Colombiana. 1991;16:213-21.
3. Forero Caballero H. Momentos Históricos de la Medicina Colombiana. Bogotá: Editorial Prismagraf; 2011.

4. Fresquet. JL. Instituto de Historia de la Ciencia y Documentación (Universidad de Valencia-CSIC). Enero, 2004. http://www.historiadelamedicina.org/vaquez.html Fecha de acceso Octubre 2 de 2017.

5. Ershler I. Willem Einthoven-the man, The string galvanometer electrocardiograph. Arch Intern Med. 1988;148:453-5.

6. Acierno LJ. The history of Cardiology. New York: Parthenon Publishing Group; 1994.

7. Fresquet Febrer JL. Willem Einthoven (1860-1921). Disponible en: http://www. historiadelamedicina.org/einthoven.html. Fecha de acceso Octubre 8 de 2017.

8. Rodríguez Gama A. Willem Einthoven "Por su descubrimiento del mecanismo del electrocardiograma'”. En: Premios Nobel de Fisiología o Medicina 1901-2016. Bogotá: Digiprint Editores, Segunda edición; 2016. p. 80. ISBN 978-958-46-9954-1.

9. Lozano G. Historia de la Cardiología en Colombia. Acta Med Col. 1989;14:41-7.

10. Otero E. Cronología Histórica Academia Nacional de Medicina. Preparada por Efraim Otero Ruiz, Presidente, con base en las notas cuidadosamente recogidas por el historiador Humberto Cáceres. https://encolombia.com/medicina/revstasmedicas/academedicina/va-24/cronologia6/2/, fecha de acceso Agosto 28 de 2017.

11. Trujillo G. Estudio clínico de un caso de endocarditis maligna de evolución lenta, (Enfermedad de Osler). Revista de la Facultad de Medicina. Universidad Nacional. 1932;1:285-91.

12. Trujillo G. Juicio crítico sobre un trabajo científico. Revista de la Facultad de Medicina. Universidad Nacional. 1936; 4:721-30.

13. Gnecco Mozo F. El trabajo del corazón en Bogotá: Introducción al estudio de la tensión arterial media en Colombia. Bogotá: Editorial Cromos; Bogotá; 1936.

14. De Zubiría R. Antonio Vargas Reyes y la medicina del siglo XIX en Colombia. Academia Nacional de Medicina. Bogotá: Editorial Academia Nacional de Medicina; 2002. p. 103-5. ISBN: 9589711758. septiembre de.

15. El Paludismo. Estudio Clínico. Revista de la Facultad de Medicina. Universidad Nacional. 1943; 11(10);585-611. 